\title{
Rugged optical mirrors for Fourier transform spectrometers operated in harsh environments
}

\author{
Dietrich G. Feist ${ }^{1}$, Sabrina G. Arnold ${ }^{1}$, Frank Hase ${ }^{2}$, and Dirk Ponge ${ }^{3}$ \\ ${ }^{1}$ Max Planck Institute for Biogeochemistry (MPI-BGC), Jena, Germany \\ ${ }^{2}$ Karlsruhe Institute of Technology (KIT), Institute of Meteorology and Climate Research (IMK-ASF), Karlsruhe, Germany \\ ${ }^{3}$ Max-Planck-Institut für Eisenforschung GmbH, Düsseldorf, Germany \\ Correspondence to: Dietrich G. Feist (dfeist@bgc-jena.mpg.de)
}

Received: 29 July 2015 - Published in Atmos. Meas. Tech. Discuss.: 20 October 2015

Revised: 11 May 2016 - Accepted: 16 May 2016 - Published: 31 May 2016

\begin{abstract}
The Total Carbon Column Observing Network (TCCON) and the Network for the Detection of Atmospheric Composition Change (NDACC) operate a number of Fourier transform spectrometers (FTSs) that measure trace gases in the atmosphere by observing solar spectra. To guide the sunlight into the FTS, a solar tracker has to be placed outside. This device needs high-quality optical mirrors with good reflectance in the near and mid-infrared.

More and more FTS stations are operated in remote locations with harsh environments. Optical mirrors are usually made for laboratory conditions and might not last very long there. At the TCCON site on Ascension Island which is operated by the Max Planck Institute for Biogeochemistry (MPIBGC), several mirrors from different optical manufacturers were destroyed within weeks.

To continue operation, the MPI-BGC had to develop rugged mirrors that could sustain the harsh conditions for months or even years. While commercially available mirrors are typically made from a substrate covered with a thin reflective coating, these rugged mirrors were made from stainless steel with no additional coating. Except for their lower reflectance (which can easily be compensated for), their optical properties are comparable to existing mirrors. However, their rugged design makes them mostly immune to corrosion and scratching. Unlike most coated mirrors, they can also be cleaned easily.
\end{abstract}

\section{Introduction}

\subsection{Atmospheric trace gas measurements with Fourier transform spectroscopy}

Many atmospheric molecules have characteristic spectral lines in the infrared region of the spectrum. When infrared radiation from the sun travels through the atmosphere, it is absorbed by molecules of trace gases in a characteristic way. By spectrally analysing the incoming sunlight on the ground, the total number of molecules in the light path - the so-called total column - can be measured. Fourier transform spectrometers (FTSs) are ideal for such measurements in the near infrared (NIR) as well as the mid-infrared (MIR). Unlike many other spectrometers, they combine large spectral bandwidth - which allows them to measure many species - with high spectral resolution that provides high accuracy.

Both the Network for the Detection of Atmospheric Composition Change (NDACC) (Kurylo and Zander, 2000) as well as the Total Carbon Column Observing Network (TCCON) (Wunch et al., 2011) have set up FTS sites all over the world (see Fig. 1). Historically, NDACC has been focused on trace gases related to stratospheric and tropospheric chemistry. TCCON's goal is to measure greenhouse gases like $\mathrm{CO}_{2}, \mathrm{CH}_{4}, \mathrm{CO}, \mathrm{N}_{2} \mathrm{O}$, and others with a precision in the subpercent range - a very ambitious target for a remote-sensing measurement. Many of the early stations were set up in the rural and urban environments of their home institutions. In the recent years, the networks have also expanded to more remote locations that are difficult to reach or have extreme environmental conditions. One example is the TCCON station that was set up by the Max Planck Institute for Biogeo- 


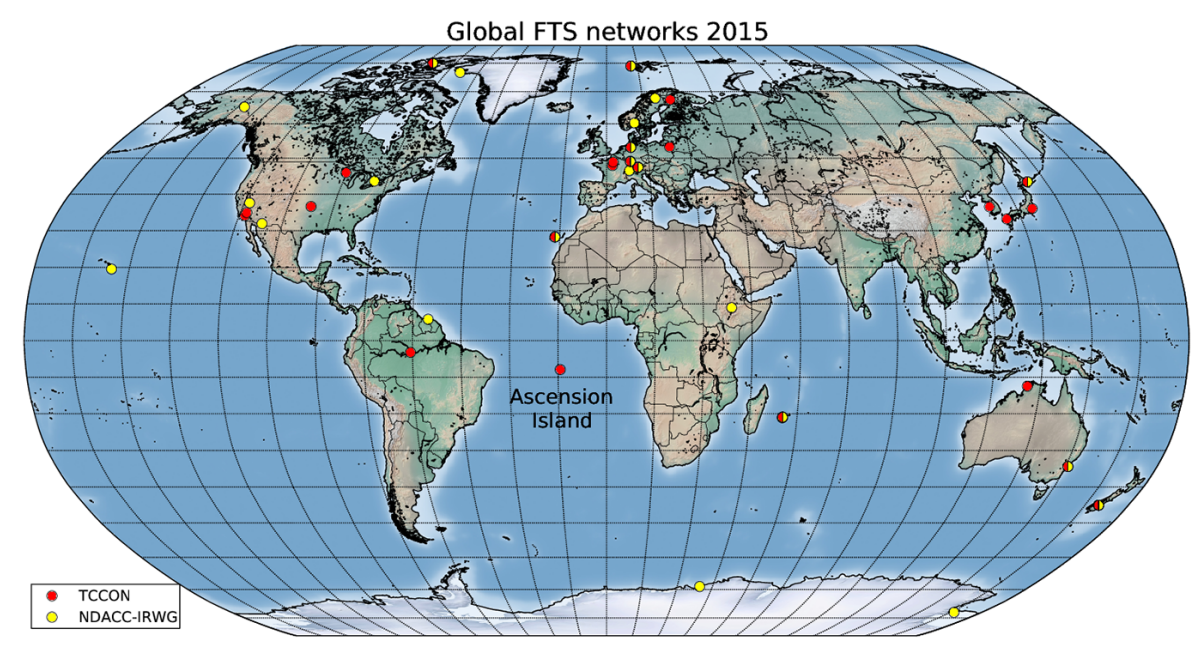

Figure 1. Map of active FTS sites in the TCCON and NDACC's Infrared Working Group (IRWG). The MPI-BGC's FTS is located on Ascension Island in the South Atlantic Ocean.

chemistry (MPI-BGC) on Ascension Island in the South Atlantic Ocean - the first equatorial station in TCCON (Geibel et al., 2010). The FTS is the same model as in other TCCON stations: an IFS125HR built by Bruker Optik GmbH in Ettlingen, Germany.

\subsection{Solar tracker and optical mirrors}

The solar tracker is the device that is responsible for guiding the sunlight into the FTS. It uses two optical mirrors that can be rotated around an azimuth and an elevation axis. During the day, the mirrors follow the sun so that the sunlight is guided straight down into the FTS that is usually located directly underneath. While the FTS itself is usually protected from the elements inside a building or container, the solar tracker has to be outside to follow the sun. It is typically mounted on a roof and is only protected from direct precipitation by a cover or dome.

Optical mirrors for this purpose are usually made from a glass substrate that provides the optically flat surface. On top of the substrate, a reflective coating is applied that provides the required high reflectance. Typical coatings are as follows.

- Unprotected gold coating: usually consisting of a single gold layer that is either vapour-deposited or sputtered. An additional adhesive layer between the substrate and the gold layer is usually needed. The advantage is the very good reflectance over a large bandwidth. The disadvantage is the soft surface that is easily scratched.

- Protected gold coating: like the unprotected gold coating but with an additional layer of $\mathrm{SiO}_{2}$ or other oxide on top of the gold layer. The advantage is that, as long as the protective layer is not damaged, the surface is much more resistant to scratches than the unprotected gold coating with similar reflective properties. The disadvantage is that it is more difficult to make and more expensive.

- Protected or unprotected aluminum coating: similar to the gold-coated mirrors except that $\mathrm{Al}$ is used as the reflective surface. The advantage is that $\mathrm{Al}$ sticks better to the substrate and can be applied without an additional adhesive layer, thus providing a more durable surface - especially with an additional protective $\mathrm{SiO}_{2}$ coating. The disadvantage is the slightly lower reflectance compared to gold.

- Dielectric coating: multiple layers with different refractive indexes. The advantage is that the layers are typically made from hard materials and provide a durable surface. The disadvantages are the limited bandwidth and difficulty to make.

So far, not much has been published about how such mirrors behave outside normal laboratory conditions. Chu et al. (2010) studied the effects of reactive gases on gold coatings and Krijger et al. (2014) have started a study on degrading effects of mirrors in space.

\section{FTS operation in harsh environments}

While the FTS itself is usually well protected inside a building or container, the solar tracker has to be outside. Even if the solar tracker is covered from direct precipitation, it will be exposed to the environmental conditions when the sky is clear and the sun is shining.

Optical components like the solar tracker mirrors are usually made for clean laboratory conditions and a limited temperature range. In a harsh environment, the equipment - and 


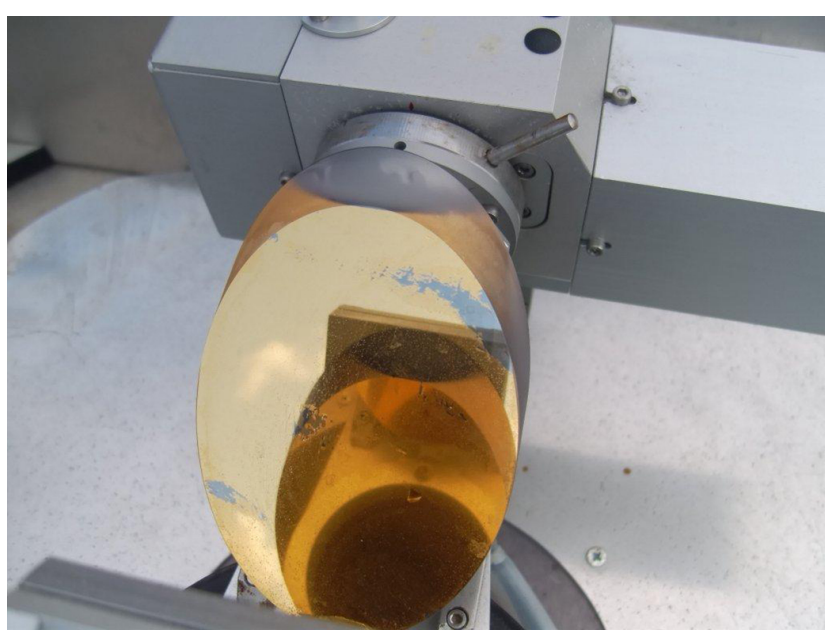

Figure 2. Surface damage on the original gold-coated mirror (Bruker) after 20 days outside and one cleaning procedure. Photo: N. John.

especially the solar tracker mirrors - can be degraded or damaged by this exposure. This effect might be slow or fast, depending on the environmental conditions.

In a harsh environment, the following effects may be encountered. They all result in a loss of mirror reflectance that would eventually disrupt the measurements.

- Dust and dirt deposition: In principle, this does not result in permanent damage if the deposited material is not corrosive. However, if the mirror surface cannot be cleaned, this deposition will build up and eventually render the mirror unusable.

- Corrosion: This is a permanent effect that degrades the mirror surface. It may be caused by the deposition of reactive substances like sea salt, often in combination with humidity or liquid water. Another reason for corrosion may be reactive gases in polluted air.

- Scratches: They also cause permanent damage to the mirror surface. Scratches may be caused by objects touching the surface or by mechanical abrasion from blowing sand or dust. They may also be the result of cleaning if the surface is not hard enough.

\subsection{Unprotected gold-coated mirror performance}

The original solar tracker mirrors by Bruker have a vapourdeposited gold coating on a glass substrate. There is no additional protective layer. In rural or urban areas, these mirrors typically last for several years. However, in the harsh environment of Ascension Island, the surface lost large parts of its coating already 3 weeks after installation (see Fig. 2). One attempt to clean the mirrors by rinsing them with distilled water and ethanol without touching the surface only did

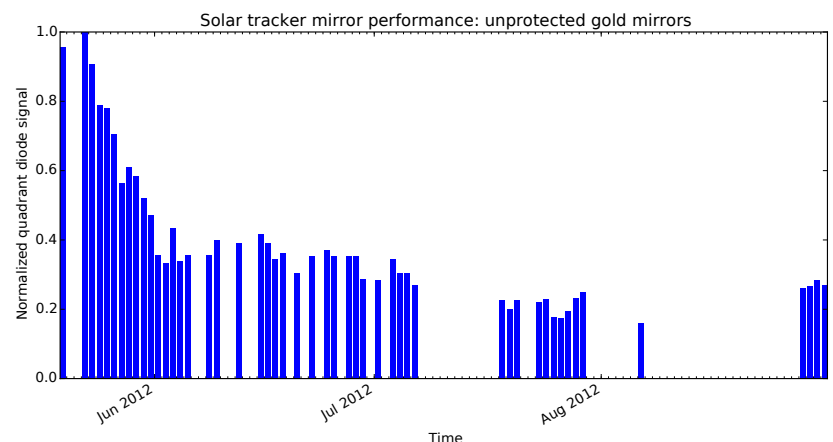

Figure 3. Reflectance loss of the original unprotected gold-coated mirrors (Bruker) on Ascension Island. The normalised maximum light intensity per day at the quadrant diode of the solar tracker is plotted as a measure of reflectance loss over time. The plot shows the combined reflectance loss for both solar tracker mirrors.

more damage. After 6 months of exposure, the gold coating was completely lost.

The problem with the coating was first noticed as a decrease of the signal of the solar tracker's quadrant diode. Figure 3 shows this normalised signal over a period of 3.5 months. The maximum signal on the quadrant diode for each day was normalised to 1 and plotted over time. The loss in signal corresponds roughly to the loss in total reflectance for the two mirrors of the solar tracker. The original Bruker mirrors lost about $80 \%$ of their combined reflectance within 2 weeks of operation on Ascension Island.

\subsection{Mirror corrosion test}

In September 2012, an empirical test of several sample mirrors was conducted. The mirrors were of different design and made by different manufacturers. They were all sent to Ascension Island and put outside for 1 month. During this time, they were exposed to sea salt spray, dust, wind, sunlight, and rain. The combined effect of constant wind and fine quartz sand might also have had an additional abrasive effect on the mirror surfaces. One half of each mirror was covered with an adhesive foil to preserve the original surface for later comparison. The results of the mirror tests are summarised in Table 1 .

Mirror no. 6 was the original mirror type with unprotected gold coating. It was sent along as a reference. During the test, its coating was lost completely. Other mirrors, namely ones with protected gold coatings, performed much better. However, it should be noted that none of the mirrors were designed for such conditions. Also, the effect of regular cleaning could not be tested this way.

\subsection{Protected gold-coated mirror performance}

Following the mirror test, a second set of mirrors with a protected gold coating was produced by Layertec. These mirrors were made in the same way as the sample mirror no. 5 in Ta- 
Table 1. Results of mirror test after 1 month of outdoor exposure on Ascension Island. Details about the mirror suppliers are provided in the acknowledgements.

\begin{tabular}{lllll}
\hline No. & Supplied by & Substrate & Surface/coating & Performance \\
\hline 1 & Bruker Optics & aluminum & galvanic gold coating & speckles and holes in coating \\
2 & Bruker Optics & glass & gold with protective layer & no visible damage \\
3 & Bruker Optics & glass & silver with protective layer & no visible damage \\
4 & Optics Balzers & glass & gold with protective layer & several small holes in coating \\
5 & Layertec & glass & gold with protective layer & no visible damage \\
6 & Bruker Optics & glass & unprotected gold coating & coating completely lost \\
7 & NA & steel (1.4301) & polished & speckles and dull areas \\
\hline
\end{tabular}

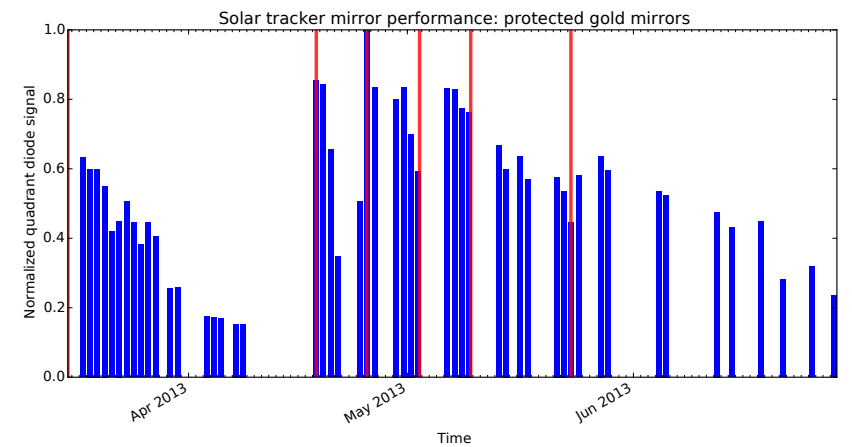

Figure 4. Reflectance loss of the protected gold-coated mirrors (Layertec), like Fig. 3. Red vertical lines indicate when the mirrors were cleaned.

ble 1 which had shown no signs of degradation during the mirror test. The new mirrors were installed in March 2013. Figure 4 shows that these mirrors also lost about $80 \%$ of their reflectance within 4 weeks of operation. However, this initial loss was most likely caused by dust deposition. By rinsing the mirrors with distilled water and ethanol, the reflectance could be restored several times (Fig. 4).

However, apparently the mirrors did not take the cleaning procedure well. After the fifth cleaning, first visible damage to the surface appeared. During the sixth cleaning procedure, large parts of the coating were destroyed. Figure 5 shows the damage to the elevation mirror. After 6 months of operation, the coating on this mirror was completely lost. On the less exposed azimuth mirror, about $50 \%$ of the coating was lost.

\section{Rugged mirrors}

A rugged mirror would have to be able to endure the conditions described in Sect. 2 without significant loss of reflectance at least over a period of several months. This period should be long enough so that mirrors only have to be exchanged during regular maintenance visits. Table 2 lists several properties that are important to achieve this goal.

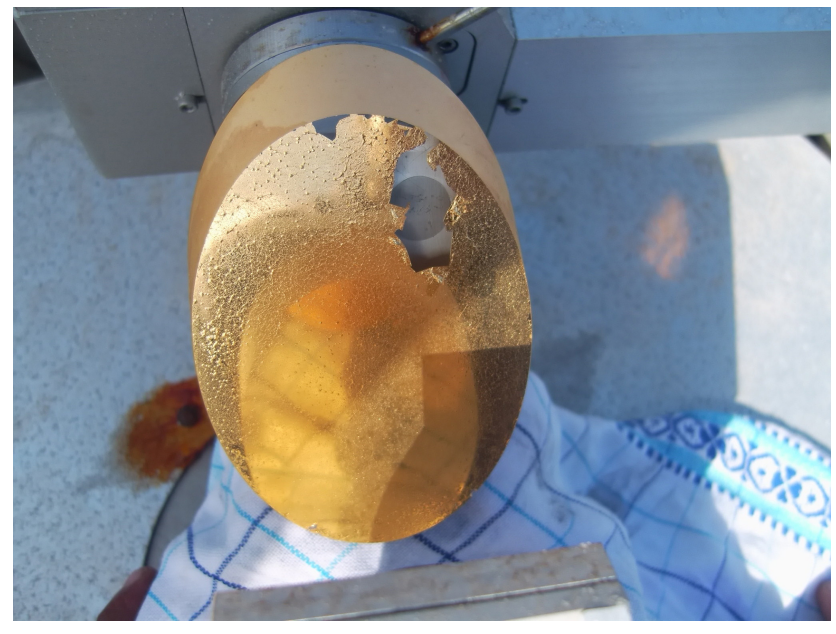

Figure 5. Surface damage on the protected gold-coated mirror (Layertec) after 70 days outside and six cleaning procedures. Photo: N. John.

\subsection{Rugged mirror material}

None of the tested coated mirrors were able to withstand the harsh environmental conditions on Ascension Island for more than a few weeks. An alternative design are mirrors made from solid material with a polished surface. Some of the considered materials were as follows.

- Aluminum: known to suffer from salt corrosion if not anodised. However, anodising and polishing are mutually exclusive.

- Chromium: very hard, very resistant to corrosion. However, it is a brittle material and therefore difficult to machine and polish.

- Silicon: not expected to suffer from corrosion, very hard surface, easy to polish. However, it only has a low reflectance of only $50 \%$ in the NIR and MIR. Since the solar tracker has two mirrors, that would result in a $75 \%$ signal loss.

- Germanium: very similar properties as silicon. 
Table 2. Design properties for rugged mirrors. The first column provides a measure of importance for the individual properties: required $(+++)$, important $(++)$, useful $(+)$.

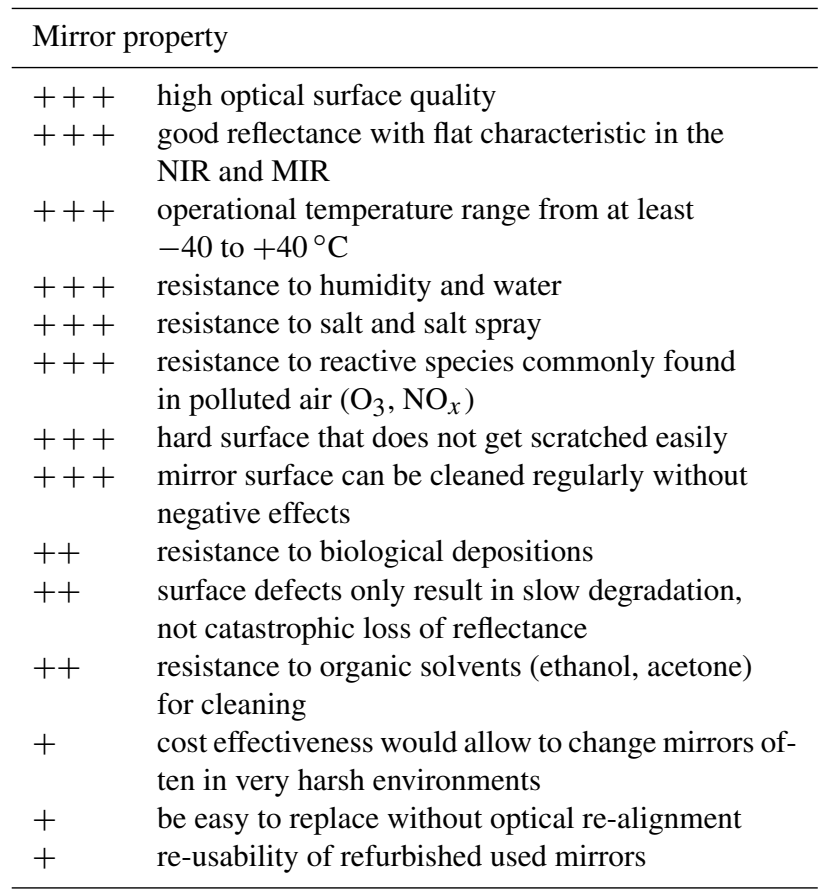

- Gold: resistant to corrosion, highly reflective in the NIR and MIR. However, too expensive for a solid mirror and too soft for polishing.

- Platinum: very resistant to corrosion and hard enough for polishing. However, even more expensive than gold.

- Stainless steel: dozens of alloys with a wide range of properties are available. Medium reflectance of $65 \%$ in the NIR and MIR had been measured for a polished steel plate of type 1.4301 (see Fig. 6) - even though this material did suffer from corrosion during the test (see Sect. 2.2).

Stainless steel appeared to be a good choice for a solid mirror material. The main issue was to select an alloy with high corrosion resistance and good surface properties for polishing.

The pitting resistance equivalent number (PREN) is a measure for the corrosion resistance of stainless steel. The higher the PREN, the more resistant the steel is against corrosion. The PREN is calculated from the mass percentages of chromium $(\mathrm{Cr})$, molybdenum $(\mathrm{Mo})$, and nitrogen $(\mathrm{N})$ in the alloy as

PREN $=1 \times \% \mathrm{Cr}+3.3 \times \% \mathrm{Mo}+16 \times \% \mathrm{~N}$.

In general, alloys with PREN $>32$ are considered to be seawater resistant (Deutsche Edelstahlwerke, 2016). Figure 6 lists PRENs for several common stainless steel types.

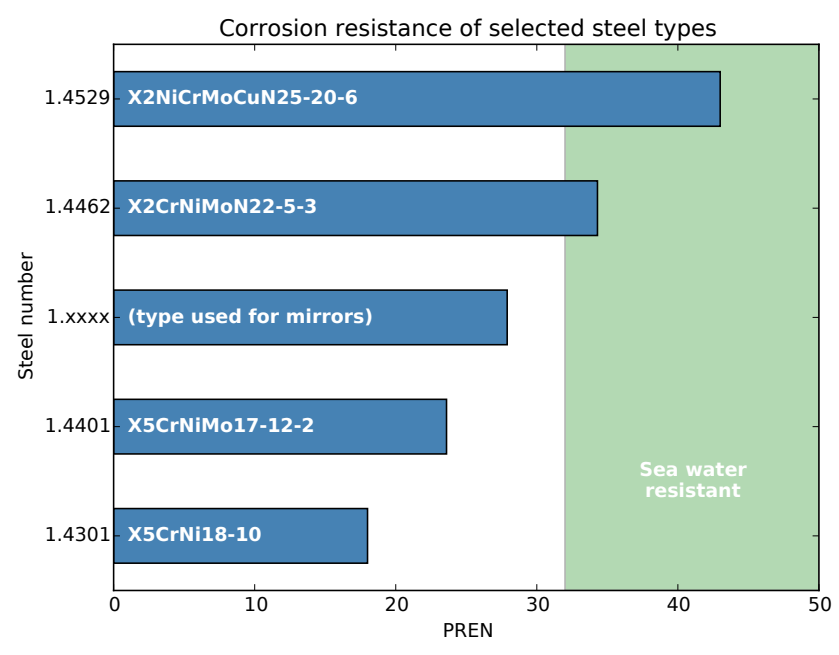

Figure 6. PREN for common stainless steel types. Steel numbers and grades are according to the European EN 10088-3 norm (European Committee for Standardization, CEN). The steel type labelled as 1.xxxx is the one that was chosen for the mirrors.

For the properties of a stainless steel mirror, the crystalline structure of the alloy is as crucial as its corrosion resistance. Stainless steel comes in different crystalline structures: ferritic, martensitic, austenitic, and duplex. Duplex stainless steels, like type 1.4462 in Fig. 6, are a mixture of austenitic and ferritic microstructure constituents. They offer very high corrosion resistance. However, because of the two intermixed crystalline structures, they also have a granular surface structure which is not suitable for a mirror surface.

For the stainless steel mirrors, an alloy that combines high corrosion resistance with excellent surface quality (labelled as 1.xxxx in Fig. 6) was chosen. It is commonly used for medical as well as industrial applications that involve high chlorine concentrations. Please note that the exact steel type cannot be provided here due to pending commercialisation of the rugged mirror design. Section 5 (Data availability) has more details on how our results can still be verified.

\subsection{Rugged mirror design}

The rugged mirrors were designed to be drop-in replacements for the existing mirrors of the Bruker A547 solar tracker (a widely used model in the TCCON and wider FTS community). The principal shape of the mirror is a disc cut out of a cylinder with a diameter of $80 \mathrm{~mm}$ at an angle of $45^{\circ}$. The mirror surface is therefore a plane ellipse with a length of $113 \mathrm{~mm}$ and a width of $80 \mathrm{~mm}$ (see Fig. 7). This is almost the same design as the original Bruker mirror, only about $1 \mathrm{~mm}$ thicker. The mirror design for azimuth and elevation mirror is identical.

The main design goal of the rugged mirrors was the same as for the original Bruker mirrors: a flatness of better than $1 \lambda$ at $633 \mathrm{~nm}$. This was verified by interferometric measurements at $460 \mathrm{~nm}$ by the company that lapped and polished the 


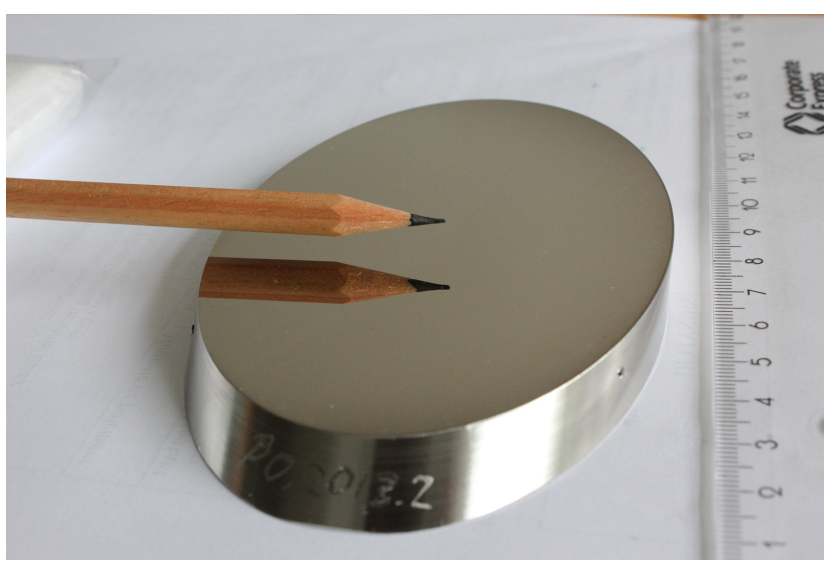

Figure 7. Stainless steel mirror with lapped and polished optical grade surface. Photo: D. Feist.

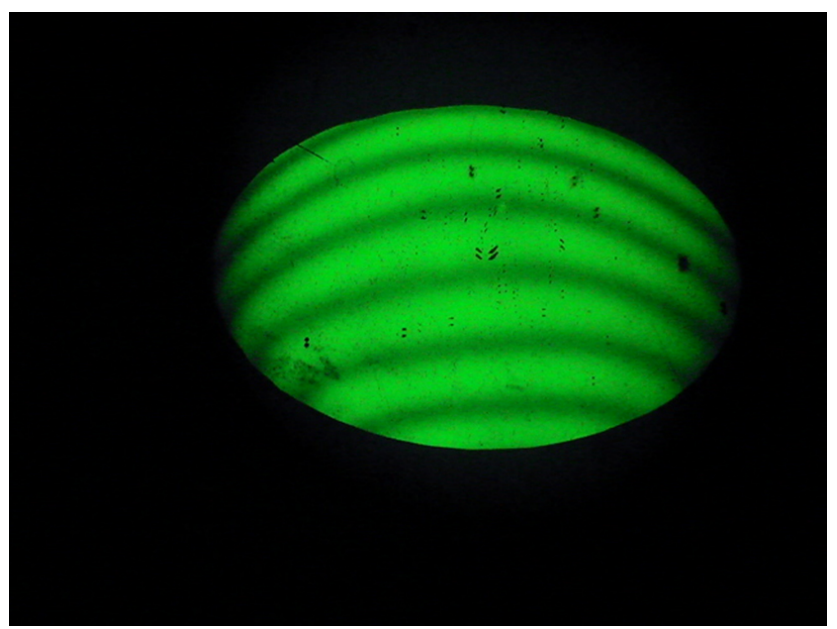

Figure 8. Interferometric test of the steel mirror's optical flatness at $\lambda=460 \mathrm{~nm}$.

steel mirrors. In Fig. 8, the pattern of parallel bright and dark lines is characteristic for a flat surface. Surface irregularities would show up as distortions of the band-shaped structures, for example as closed lines that surround a local minimum or maximum.

\subsection{Reflectance}

The second goal was a flat reflectance response with no mirror-induced artifacts from the MIR to the NIR. This was verified using a Bruker IFS125HR FTS at the Karlsruhe Institute of Technology. A globar was used as the light source to cover the MIR to the NIR. This way, a spectral range of 1800 to $12000 \mathrm{~cm}^{-1}$ could be covered. The reflectance was measured by comparing a spectrum acquired from the direct light path to a spectrum acquired after a reflection on both mirrors.

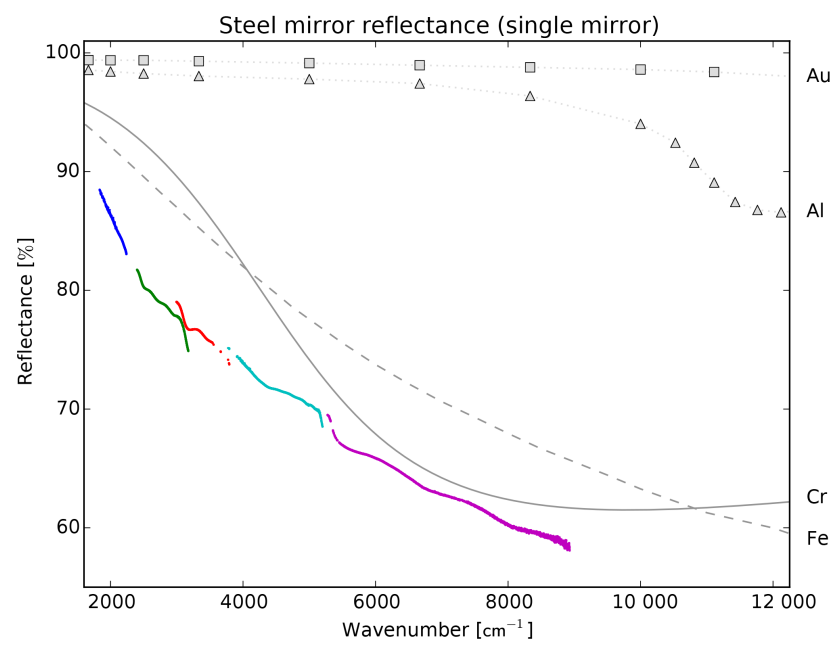

Figure 9. Measurements of the steel mirrors' reflectance $R$, normalised for a single-mirror reflection (coloured lines). The different colours correspond to different optical filters that were used. Spectral windows with significant absorption from $\mathrm{H}_{2} \mathrm{O}$ and $\mathrm{CO}_{2}$ were blanked out. Measured reflectance values for $\mathrm{Au}$ and $\mathrm{Al}$ as well as modelled values for $\mathrm{Cr}$ and $\mathrm{Fe}$ are shown for comparison.

To simulate the setup of the solar tracker, each mirror was oriented at an incident angle of $45^{\circ}$ to the incoming light. The double reflection also produced a stronger effect. Figure 9 shows the resulting reflectance $R$ which was normalised for a single-mirror reflection. In other words, with two mirrors having reflectances of $R_{1}$ and $R_{2}$, respectively, the normalised reflectance is

$R=\sqrt{R_{1} R_{2}}$.

In Fig. 9, the different colours correspond to the standard NDACC bandpass filters that were used during the measurement. For each filter, only the spectral band where the filter transmission was above $25 \%$ was plotted. To reduce artifacts from the ambient air, spectral line data for $\mathrm{H}_{2} \mathrm{O}$ and $\mathrm{CO}_{2}$ from the HITRAN 2012 database (Rothman et al., 2013) were used to calculate the transmission of a $1 \mathrm{~m}$ path through ambient air. Regions with less than $99 \%$ transmission were blanked out.

For comparison, the plot also shows reflectance curves for the common mirror coating materials Au (Bennett and Ashley, 1965) and $\mathrm{Al}$ (Bennett et al., 1963) from experimental data. The other two reflectance curves were derived from theoretical calculations for the two main components of stainless steel: Fe (Ordal et al., 1988) and Cr (Rakić et al., 1998). The actual data for the calculation of the respective complex refractive indexes were provided by Polyanskiy (2016).

The main result was that the steel mirrors showed a relatively low but steady reflectance in the range of $60 \%$ in the NIR to almost $90 \%$ in the MIR. The measured reflectance curve looked qualitatively similar to what one would expect from the theoretical reflectance curves of $\mathrm{Fe}$ and $\mathrm{Cr}$. 
Table 3. Spectra used in the field intercomparisons in Sect. 4. Mirror age is time since mirror installation for $S_{1}, S_{2}$, and $S_{3}$ and time since last mirror cleaning for $\mathrm{S}_{4}$ and $\mathrm{S}_{5}$.

\begin{tabular}{lllrrr}
\hline Spectrum & Timestamp (UTC) & Mirror type & Mirror age & Solar zenith angle & $\mathrm{XH}_{2} \mathrm{O}$ \\
\hline $\mathrm{S}_{1}$ & 22 May 2012, 12:10:22 & unprotected gold & 0 days & $30.4^{\circ}$ & $3837 \mathrm{ppm}$ \\
$\mathrm{S}_{2}$ & 30 May 2012, 12:34:44 & unprotected gold & 8 days & $30.2^{\circ}$ & $3730 \mathrm{ppm}$ \\
$\mathrm{S}_{3}$ & 28 August 2012, 14:37:26 & unprotected gold & 98 days & $30.1^{\circ}$ & $3997 \mathrm{ppm}$ \\
$\mathrm{S}_{4}$ & 24 November 2013, 14:36:46 & stainless steel & 0 days & $30.0^{\circ}$ & $3725 \mathrm{ppm}$ \\
$\mathrm{S}_{5}$ & 13 December 2013, 11:01:29 & stainless steel & 18 days & $30.5^{\circ}$ & $4307 \mathrm{ppm}$ \\
\hline
\end{tabular}

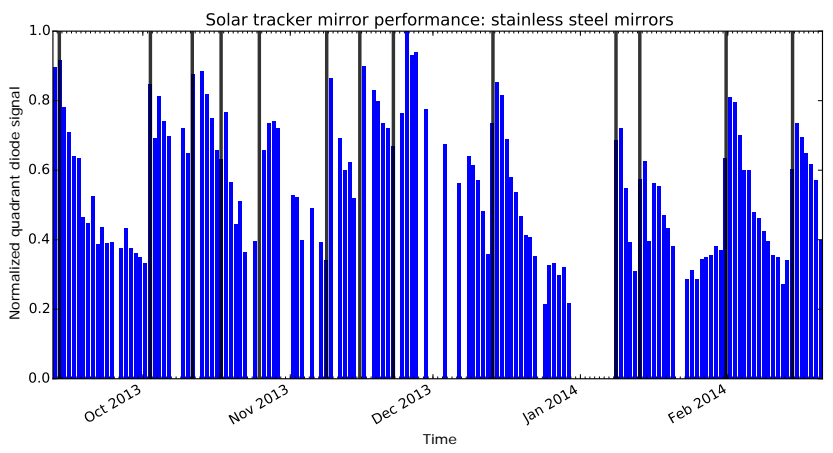

Figure 10. Reflectance over time for the first set of stainless mirrors (MPI-BGC). Like in Fig. 4, the red vertical lines indicate when the mirrors were cleaned.

Note that despite the removal of ambient-air absorption features, there are strongly absorbing spectral lines of $\mathrm{H}_{2} \mathrm{O}$ and $\mathrm{CO}_{2}$ near several of the filter edges. So the kinks near the overlapping filter edges at 3000 and $5300 \mathrm{~cm}^{-1}$ are most likely spectral artifacts and not actual effects of the steel mirrors. It took several minutes to collect the spectra and then change the experimental setup from direct light path to double-mirror reflection. It is likely that - with three people in a relatively small laboratory container - the $\mathrm{H}_{2} \mathrm{O}$ and $\mathrm{CO}_{2}$ concentration in the ambient air changed significantly during that time. This would have caused offsets in the measured spectra near $\mathrm{H}_{2} \mathrm{O}$ and $\mathrm{CO}_{2}$ spectral lines and thus artifacts in the calculated reflectance. Since all NDACC and TCCON retrieval windows are well away from the filter edges, the kinks should not affect the retrievals in any way - independent of their cause.

\subsection{Endurance in harsh environments}

The MPI-BGC stainless steel mirrors were installed during a maintenance visit in August/September 2013. Compared to the coated mirrors, their reflectance was considerably lower from the beginning. This had to be compensated by adjusting the gain of the quadrant diode and opening the input iris aperture of the FTS by about $40 \%$.

These mirrors also showed a quick reflectance loss due to dust deposition (Fig. 10). However, their reflectance could be restored by cleaning without noticeable surface damage.
For the cleaning, the mirrors were simply wiped with a damp microfibre cloth. Even after 6 months of operation, the most exposed elevation mirror showed only a few dull spots on the surface. A weak pattern of scratches perpendicular to the direction of cleaning was probably caused by blowing sand. The other mirror showed no obvious surface defects. After 6 months of operation, the total reflectance of the two-mirror system (after cleaning) had not changed significantly.

\section{Field intercomparison of gold-coated and stainless steel mirrors}

A direct intercomparison of the different mirror types under laboratory conditions was never possible. Every time a new set of mirrors was installed at the Ascension Island station, the previous setup had already been destroyed. However, some of the properties of the different mirror types could later be derived from the observation time series. To make these observations comparable, only spectra from a narrow range of solar zenith angles $\left(30 \pm 0.5^{\circ}\right)$ and with comparable atmospheric humidity (3500-4500 ppm) were selected. Only a small number of spectra matched these selection criteria. Matching spectra could be found for the original unprotected gold mirrors as well as the stainless steel mirrors but not for the protected gold mirrors.

Table 3 lists the spectra that were used in the following comparisons. In this table, mirror age is the time since mirror installation for the gold-coated mirrors and time since the last cleaning for the stainless steel mirrors. Because of the atmospheric humidity criterion, most spectra were from a very small selection of measurement days. Therefore the possible choices with respect to mirror age were very limited.

\subsection{Signal loss over time and effect on signal-to-noise ratio (SNR)}

Figure 11 shows spectra measured with the original unprotected gold mirrors as well as the first set of stainless steel mirrors. The spectral amplitude was normalised to the outof-band noise level to provide an estimate of SNR and thus make the spectral amplitudes comparable. Note that the SNR for the stainless steel mirror was slightly higher because the 

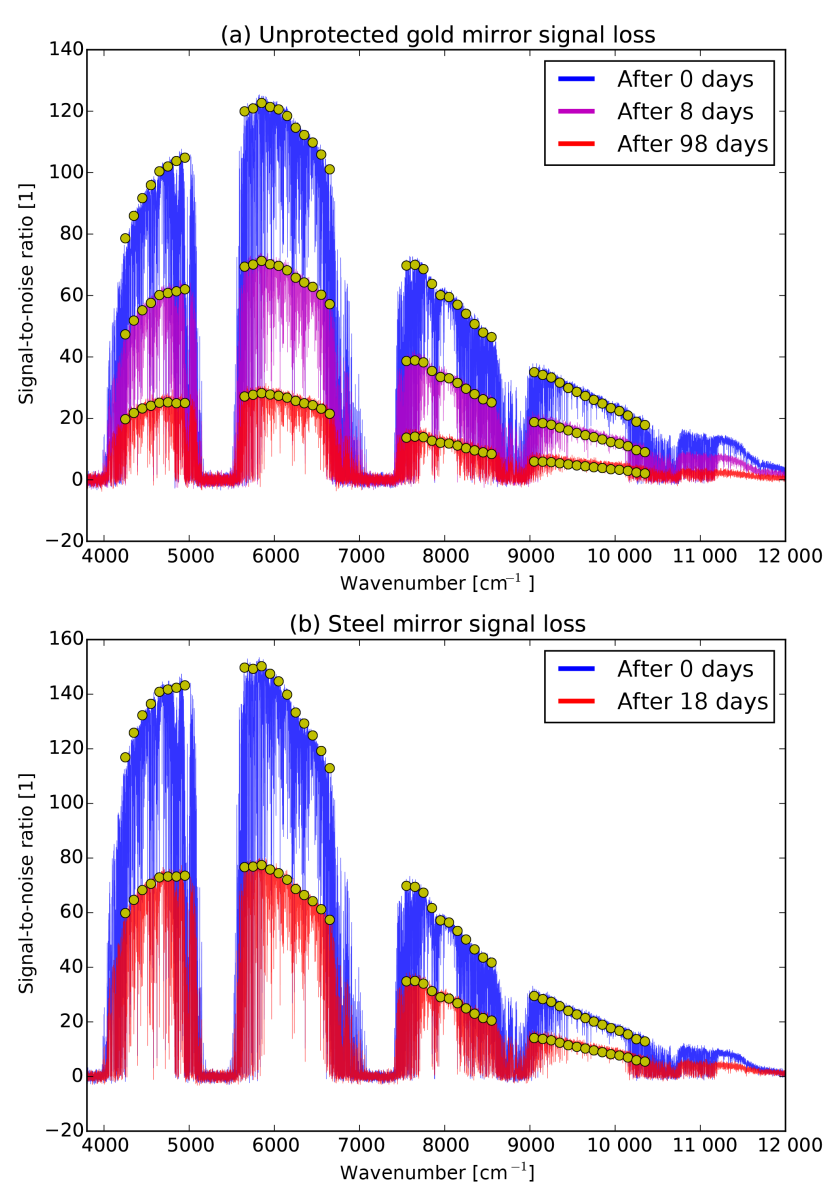

Figure 11. Measured field spectra and the effect of signal loss over time. (a) For the original gold-coated mirrors (blue: $\mathrm{S}_{1}$; magenta: $S_{2}$; red: $S_{3}$ from Table 3). (b) For the stainless steel mirrors (blue: $\mathrm{S}_{4}$; red: $\mathrm{S}_{5}$ from Table 3 ). The spectrum amplitude was normalised with the out-of-band noise level to provide an estimate of signal-tonoise ratio. The yellow circles are reference points located roughly in the centre of the spectra's upper noise envelope and well away from strong absorption lines. The time in the legend is the mirror age as defined in Table 3.

FTS's input aperture was increased to compensate for the steel mirrors' lower reflectance.

For both mirror types, the SNR degraded quickly already after a few days of exposure. At the end of the measurement period, the signal loss for the gold-coated mirror was in the order of $80 \%$. The stainless steel mirror also lost signal quickly. However, this loss was only due to dust deposition and could be restored by cleaning the mirror.

\subsection{Frequency-dependent effects of mirror degradation}

The mirror degradation also affected the mirrors' frequency response. Figure 12 shows that this effect was relatively weak for the stainless steel mirrors. The signal loss was about $15 \%$ stronger for shorter wavelengths. This is not surprising as

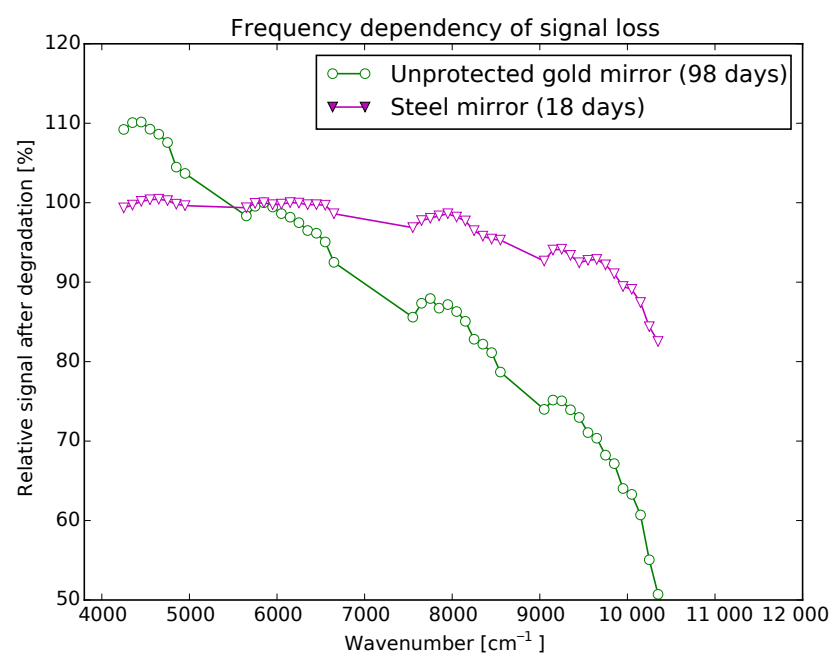

Figure 12. Effect of mirror degradation on frequency response at the reference points defined in Fig. 11. The green circles were derived from $S_{3} / S_{1}$ and the magenta triangles from $S_{5} / S_{4}$ (from Table 3).

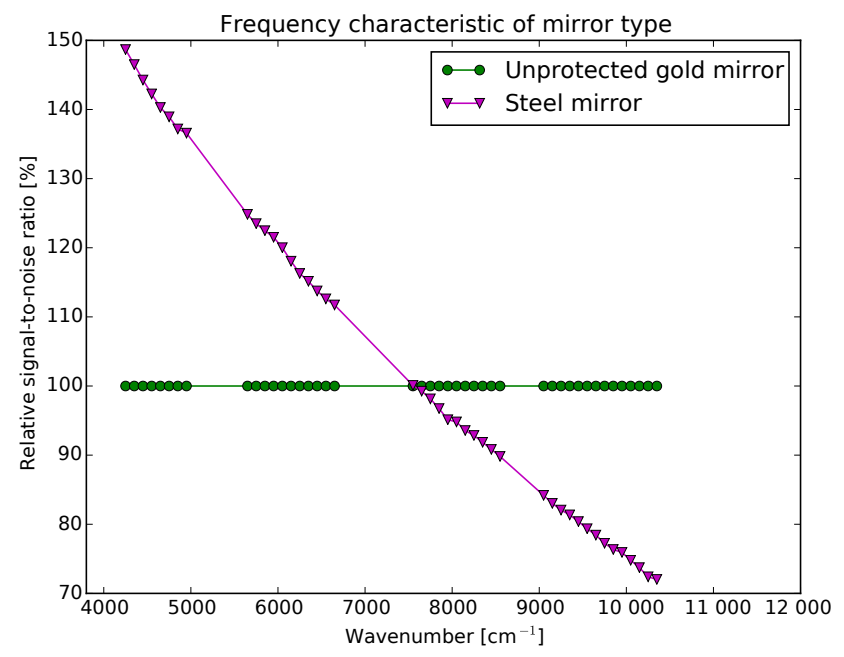

Figure 13. Frequency characteristic of the stainless steel mirror evaluated at the reference points defined in Fig. 11. The frequency response was normalised with respect to the original gold-coated mirror shortly after installation. Thus the green circle were derived from $S_{1} / S_{1}$ and the magenta triangles from $S_{4} / S_{1}$ (from Table 3).

the dust deposition would mainly reduce the mirror's surface quality temporarily. This should have a greater effect at shorter wavelengths.

For the gold-coated mirror, the frequency effect as shown in Fig. 12 was much stronger. This was probably not just an effect of the dust deposition but rather of the removal of the gold coating. Eventually, the largest part of the gold coating was removed and the reflecting surface was only provided by the glass substrate - with very different optical characteristics. 


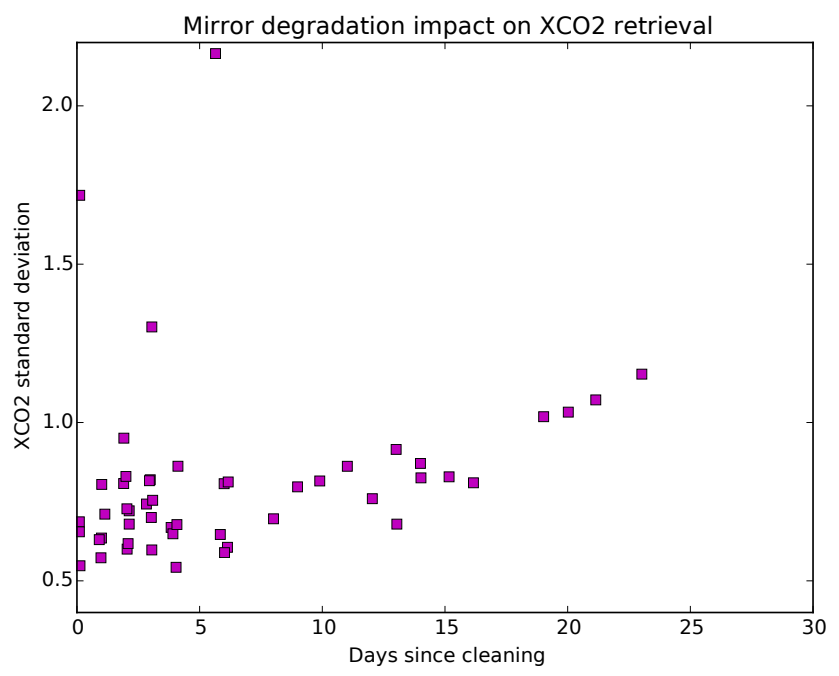

Figure 14. Impact of dust-related mirror degradation on retrieval quality. The standard deviation of at least $30 \mathrm{XCO}_{2}$ observations from the same day in a solar zenith angle range of $30 \pm 5^{\circ}$ was plotted over the number of days since the last cleaning of the stainless steel mirrors.

The selected spectra also allowed comparing the frequency characteristics of the unprotected gold-coated mirror with the stainless steel mirrors - even though they were installed more than 14 months apart. In Fig. 13, the stainless steel mirror's frequency response was normalised with respect to the goldcoated mirrors (divided by $\mathrm{S}_{1}$ ). The resulting relative frequency response shows qualitatively similar spectral characteristics as the reflectance derived from the laboratory measurements in Fig. 9.

\subsection{Impact of mirror degradation on the retrieval}

The most critical question was if and how the mirror degradation would affect the data quality of the retrieved parameters like the dry-air mole fraction of $\mathrm{CO}_{2}$ (referred to as $\mathrm{XCO}_{2}$ ) (Wunch et al., 2011). This was estimated by looking at the standard deviation of consecutive $\mathrm{XCO}_{2}$ observations from the same day which were taken in a comparable solar zenith angle range of $30 \pm 5^{\circ}$. Under the assumption that the actual $\mathrm{XCO}_{2}$ of the atmosphere had remained constant, the standard deviation of the retrieved $\mathrm{XCO}_{2}$ was used as an indicator for the quality of the retrieval. This assumption is reasonable on Ascension Island, where $\mathrm{XCO}_{2}$ is dominated by long-range transport and local $\mathrm{CO}_{2}$ sources and sinks are negligible.

This quality indicator was analysed for the stainless steel mirrors in order to find out the optimal cleaning interval. There were not enough data from the two gold-coated mirrors to clearly distinguish changes in the $\mathrm{XCO}_{2}$ standard deviation due to mirror degradation from other effects like clouds. Figure 14 shows that the $\mathrm{XCO}_{2}$ standard deviation correlates with the number of days since the mirror was last cleaned. With the exception of a few outliers - which are probably not related to the mirror state - there is no obvious correlation between $\mathrm{XCO}_{2}$ standard deviation and the number of days since the last cleaning during the first 10 days.

After more than 15 days since the last cleaning, the $\mathrm{XCO}_{2}$ standard deviation clearly rises with every additional day and reaches values above $1 \mathrm{ppm}$ after more than 19 days. This was only a qualitative result due to the limited amount of observations that were made with mirrors that had not been cleaned for more than 2 weeks. However, it gave a clear indication that the mirrors should be cleaned at least every 2 weeks to minimise impact on the retrieval. Eventually, a weekly cleaning schedule was set up with the local technical support on Ascension Island.

\subsection{Potential impact of nonlinear mirror frequency response on the retrieval}

Kiel et al. (2016) recently reported on the effects that nonlinearities in the FTS's frequency response could have on the retrieved dry-air mole fractions with TCCON's current retrieval code GGG2014 (Wunch et al., 2015). The TCCON instrument at Karlsruhe, Germany, uses a nonstandard optical setup which has a more nonlinear frequency response than other TCCON instruments. The resulting nonlinear continuum term in the spectra affects the retrieval in wide microwindows like the one used for retrieving $\mathrm{XO}_{2}$. Since the current GGG2014 code can only fit a linear continuum term to the spectrum, nonlinear effects reduce the quality of the spectral fit and lead to a bias in the order of almost $1 \mathrm{ppm}$ for $\mathrm{XCO}_{2}$ for the Karlsruhe instrument (Kiel et al., 2016). The effect can be compensated by using a higher-order continuum fit which might be implemented in a future GGG version.

In principle, such a nonlinear spectral continuum term could also be produced by the solar tracker mirrors. The original gold-coated mirrors are not critical in this respect due to their very flat reflectance curve. However, the gradient in the stainless steel mirrors' reflectance curve (Figs. 9 and 13) could affect the retrieval in principle. A practical way to check this was to look at the spectral residuals - the difference between the measured spectrum and the fitted modelled spectrum. If all is well, the residuals should be Gaussian noise bands with no structure. Otherwise, there would be visible systematic structures in the residuals - like the ones reported by Kiel et al. (2016, their Figs. 2 and 3).

Figure 15 shows the residuals for the $\mathrm{XO}_{2}$ retrieval microwindow around $7885 \mathrm{~cm}^{-1}$. This microwindow was chosen because it covers the widest spectral range $\left(240 \mathrm{~cm}^{-1}\right)$ in the TCCON retrieval setup and should therefore be most sensitive to nonlinear continuum terms. The residuals for both the gold-coated as well as the steel mirrors showed random noise bands with similar spread and no visible structure. There was no indication that the quality of the spectral fit for the steel mirrors was lower than the one for the gold-coated mirrors. This was also verified for the other wide 


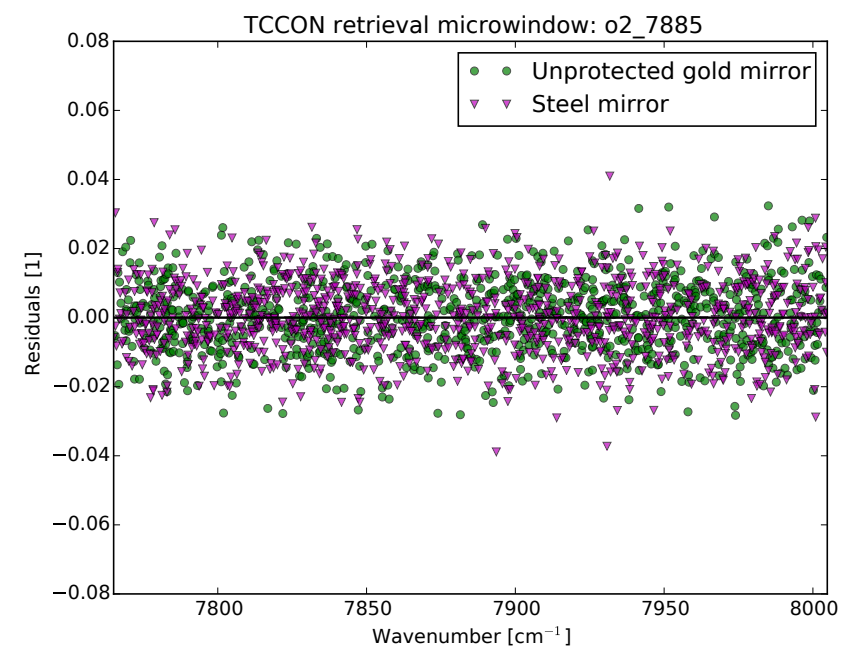

Figure 15. Spectral residuals in the $\mathrm{O}_{2}$ retrieval microwindow around $7885 \mathrm{~cm}^{-1}$ that were derived from spectra $\mathrm{S}_{1}$ (gold mirror) and $\mathrm{S}_{4}$ (steel mirror) in Table 3. This microwindow was chosen because it covers the widest spectral range in the standard TCCON retrieval. Nonlinearities in the stainless steel mirrors' frequency response should have the largest effect in this microwindow. For better visibility, only a subset of 1000 randomly chosen data points (instead of more than 30000 ) was plotted for each mirror type.

( $>20 \mathrm{~cm}^{-1}$ ) TCCON microwindows (not shown here). None of them showed any visible differences between the mirror types.

\section{Conclusions}

All coated mirrors that were tested on Ascension Island were heavily damaged after weeks or at most a few months of exposure. No coated mirror was able to be used for a period of 6 months, which would be the typical maintenance interval for the station.

The steel mirrors developed by MPI-BGC endured the harsh conditions for 6 months with only a minor loss of reflectance and no major surface damage. The mirrors were easy to clean and were not adversely affected by the procedure in any way.

The first set of stainless steel mirrors was replaced during the third maintenance visit to Ascension Island in March 2014. By that time, the mirrors had been exposed to the harsh environment for about 6 months. More than 7500 spectra had been measured during this time period. The mirrors were replaced despite the fact that they showed only minor surface defects. By now the second set of steel mirrors has already been outside for more than 18 months without obvious degradation.

The lower reflectance of the stainless steel mirrors was not a problem for the FTS on Ascension Island. The dynamic range of the instrument was large enough to compensate for the lower signal. By adjusting the input aperture slightly, the stainless steel mirrors reached an SNR level comparable to the original gold-coated mirrors.

The frequency-dependent gradient in the stainless steel mirrors' reflectance curve did not affect the retrieval quality. The spectral residuals derived from measurements with the stainless steel mirrors were indistinguishable from the ones produced with the gold-coated mirrors.

On Ascension Island, the stainless steel mirrors' reflectance degrades slowly due to dust deposition. The effect only causes problems after several weeks of exposure and can be remedied by regular cleaning. A weekly cleaning interval seemed to be sufficient to avoid adverse effects on the retrieval quality.

The rugged mirrors designed by MPI-BGC have proved to be a useful drop-in replacement for the existing mirror options of the Bruker A547 solar tracker. Because of their ruggedness, they are most likely the only viable option for harsh environments - especially in places with salt corrosion. Currently, they are also the only mirrors that allow regular cleaning. Their main disadvantage, the lower reflectance, would most likely be a problem only at high-latitude sites with very low solar intensity.

It should be straightforward to adapt these rugged mirrors for other solar trackers that are currently used in the FTS community, especially the newer ones that already use metal mirrors in favour of coated glass mirrors.

\section{Data availability}

As mentioned in Sect. 3.1, the exact steel type of the rugged mirrors cannot be disclosed here due to the pending commercialisation of the rugged mirrors. However, the far greater limitation for third parties would be to produce the optical quality surface of our mirrors. To allow others to verify our results, the MPI-BGC would lend a sample mirror to interested parties for a charge that covers the transport costs.

Figures 9, 11, 12, and 13 were derived from rather large high-resolution data files in the proprietary OPUS format used by the Bruker FTSs. These files are currently not publicly archived in the TCCON community. Upon request, the corresponding author will provide spectral data converted to NetCDF 4.

Figure 14 was generated from the publicly available TCCON data for Ascension Island (Feist et al., 2014).

Author contributions. D. G. Feist designed the stainless steel mirrors and wrote the manuscript. S. G. Arnold conducted the mirror tests on Ascension Island and analysed the effects of mirror degradation on the retrieval. F. Hase advised on the mirror design and conducted the mirror reflectance tests at KIT. D. Ponge provided essential guidance on steel corrosion mechanisms which eventually led to the selection of the optimal steel type. 
Acknowledgements. The development of the rugged solar tracker mirrors was funded by the Max Planck Society. On-site photos were taken by Nicholas John, ESA Ariane Station, North East Bay, Ascension Island. Sample mirrors for the outdoor mirror test were provided by (1) Axel Keens, Bruker Optik GmbH, Ettlingen, Germany; (2) Armin Rupp, Optics Balzers GmbH, Eschborn, Germany; and (3) Georg Böttcher and Silvia Bark-Zollmann, Layertec $\mathrm{GmbH}$, Mellingen, Germany. We would especially like to thank Ingrid Jung, Böhler-Uddeholm Deutschland GmbH, Düsseldorf, Germany, for providing good advice as well as a number of stainless steel samples that helped greatly in selecting the optimal steel type for the mirrors. The initial manuscript was written and submitted during a sabbatical visit to the Department of Physics at the University of Toronto, Canada, in the summer of 2015.

The article processing charges for this open-access publication were covered by the Max Planck Society.

Edited by: D. Brunner

\section{References}

Bennett, H. E., Silver, M., and Ashley, E. J.: Infrared Reflectance of Aluminum Evaporated in Ultra-High Vacuum, J. Opt. Soc. Am., 53, 1089-1095, doi:10.1364/JOSA.53.001089, 1963.

Bennett, J. M. and Ashley, E. J.: Infrared Reflectance and Emittance of Silver and Gold Evaporated in Ultrahigh Vacuum, Appl. Opt., 4, 221-224, doi:10.1364/AO.4.000221, 1965.

Chu, C.-T., Alaan, D. R., and Taylor, D. P.: Accelerated atmospheric corrosion testing of electroplated gold mirror coatings, in: Proc. SPIE 7786, Current Developments in Lens Design and Optical Engineering XI; and Advances in Thin Film Coatings VI, edited by: Johnson, R. B., Mahajan, V. N., and Thibault, S., vol. 7786 of Proc. SPIE, SPIE, San Diego, California, USA, 77860J, doi:10.1117/12.858779, 2010.

Deutsche Edelstahlwerke: Schematic "family tree" of the austenitic stainless steels, http://www.dew-stahl.com/fileadmin/files/ dew-stahl.com/documents/Publikationen/Werkstoffdiagramme/ 05_austenitische_Staehle_en.pdf, last access: 7 April, 2016.

European Committee for Standardization (CEN): Stainless steels Part 3: Technical delivery conditions for semi-finished products, bars, rods, wire, sections and bright products of corrosion resisting steels for general purposes, Tech. Rep. EN 10088-3:2014, CEN-CENELEC Management Centre, Brussels, Belgium, 2014.

Feist, D. G., Arnold, S. G., John, N., and Geibel, M. C.: TCCON data from Ascension Island, Saint Helena, Ascension and Tristan da Cunha, Release GGG2014R0, TCCON data archive, hosted by the Carbon Dioxide Information Analysis Center, Oak Ridge National Laboratory, Oak Ridge, Tennessee, USA, doi:10.14291/tccon.ggg2014.ascension01.R0/1149285, 2014.
Geibel, M. C., Gerbig, C., and Feist, D. G.: A new fully automated FTIR system for total column measurements of greenhouse gases, Atmos. Meas. Tech., 3, 1363-1375, doi:10.5194/amt-31363-2010, 2010.

Kiel, M., Wunch, D., Wennberg, P. O., Toon, G. C., Hase, F., and Blumenstock, T.: Improved retrieval of gas abundances from near-infrared solar FTIR spectra measured at the Karlsruhe TCCON station, Atmos. Meas. Tech., 9, 669-682, doi:10.5194/amt9-669-2016, 2016.

Krijger, J. M., Snel, R., van Harten, G., Rietjens, J. H. H., and Aben, I.: Mirror contamination in space I: mirror modelling, Atmos. Meas. Tech., 7, 3387-3398, doi:10.5194/amt-7-3387-2014, 2014.

Kurylo, M. J. and Zander, R.: The NDSC - its status after 10 years of operation, in: Proc. XIX Quadrennial Ozone Symposium, Hokkaido University, Sapporo, Japan, 167-168, 2000.

Ordal, M. A., Bell, R. J., Alexander, R. W., Newquist, L. A., and Querry, M. R.: Optical properties of Al, Fe, Ti, Ta, W, and Mo at submillimeter wavelengths, Appl. Opt., 27, 1203-1209, doi:10.1364/AO.27.001203, 1988.

Polyanskiy, M. N.: Refractive index database, available at: http:// refractiveindex.info, last access: 19 February, 2016.

Rakić, A. D., Djurišić, A. B., Elazar, J. M., and Majewski, M. L.: Optical properties of metallic films for verticalcavity optoelectronic devices, Appl. Opt., 37, 5271-5283, doi:10.1364/AO.37.005271, 1998.

Rothman, L., Gordon, I., Babikov, Y., Barbe, A., Benner, D. C., Bernath, P., Birk, M., Bizzocchi, L., Boudon, V., Brown, L., Campargue, A., Chance, K., Cohen, E., Coudert, L., Devi, V., Drouin, B., Fayt, A., Flaud, J.-M., Gamache, R., Harrison, J., Hartmann, J.-M., Hill, C., Hodges, J., Jacquemart, D., Jolly, A., Lamouroux, J., Roy, R. L., Li, G., Long, D., Lyulin, O., Mackie, C., Massie, S., Mikhailenko, S., Müller, H., Naumenko, O., Nikitin, A., Orphal, J., Perevalov, V., Perrin, A., Polovtseva, E., Richard, C., Smith, M., Starikova, E., Sung, K., Tashkun, S., Tennyson, J., Toon, G., Tyuterev, V., and Wagner, G.: The HITRAN2012 molecular spectroscopic database, 40, 450, doi:10.1016/j.jqsrt.2013.07.002, 2013.

Wunch, D., Toon, G. C., Blavier, J.-F. L., Washenfelder, R., Notholt, J., Connor, B. J., Griffith, D. W. T., Sherlock, V., and Wennberg, P. O.: The Total Carbon Column Observing Network, Phil. Trans. R. Soc. A, 369, 2087-2112, doi:10.1098/rsta.2010.0240, 2011.

Wunch, D., Toon, G. C., Sherlock, V., Deutscher, N. M., Liu, C., Feist, D. G., and Wennberg, P. O.: The Total Carbon Column Observing Network's GGG2014 Data Version, doi:10.14291/tccon.ggg2014.documentation.R0/1221662, 2015. 